\title{
Extracting the bulk viscosity of the quark-gluon plasma
}

\author{
Jean-Bernard Rose ${ }^{\mathrm{a}}$, Jean-Francois Paquet ${ }^{\mathrm{a}}$, Gabriel S. Denicol ${ }^{\mathrm{a}}$, Matthew Luzum ${ }^{\mathrm{a}, \mathrm{b}}$, \\ Bjoern Schenkec, Sangyong Jeon ${ }^{\mathrm{a}}$, Charles Gale ${ }^{\mathrm{a}}$ \\ ${ }^{a}$ Department of Physics, McGill University, 3600 rue University, Montréal, Québec H3A 2T8, Canada \\ ${ }^{b}$ Lawrence Berkeley National Laboratory, Berkeley, CA 94720, USA \\ ${ }^{c}$ Physics Department, Brookhaven National Lab, Building 510A, Upton, NY, 11973, USA
}

\begin{abstract}
We investigate the implications of a nonzero bulk viscosity coefficient on the azimuthal momentum anisotropy of ultracentral relativistic heavy ion collisions at the Large Hadron Collider. We find that, with IP-Glasma initial conditions, a finite bulk viscosity coefficient leads to a better description of the flow harmonics in ultracentral collisions. We then extract optimal values of bulk and shear viscosity coefficients that provide the best agreement with flow harmonic coefficients data in this centrality class.
\end{abstract}

\section{Introduction}

Currently, most fluid-dynamical simulations of relativistic heavy ion collisions take into account only dissipative effects originating from shear viscosity. However, QCD is a nonconformal field theory and in principle there is no a priori reason to neglect the effects of bulk viscous pressure. In this contribution, we explore the phenomenological implications of a nonzero bulk viscosity coefficient on the azimuthal momentum anisotropy of ultracentral heavy ion collisions at the Large Hadron Collider (LHC). We then extract optimal values of bulk and shear viscosity coefficients that are able to describe the flow harmonic coefficients in this centrality class. We find that, with IP-Glasma initial conditions, a finite bulk viscosity coefficient improves the description of flow harmonics in ultracentral collisions.

\section{Fluid-dynamical model of the collision}

In this work we simulate ultrarelativistic heavy ion collisions in the $0-1 \%$ centrality class at LHC energies. The initial energy density profile is obtained using the IP-Glasma initial condition model, with a thermalization time of $\tau_{0}=0.4 \mathrm{fm}$ [1]. The time evolution of this system is determined using relativistic dissipative fluid dynamics, with the main equations of motion being the continuity equation for the energy-momentum tensor, $T^{\mu v}, \partial_{\mu} T^{\mu v}=0$, where $T^{\mu \nu}=\varepsilon u^{\mu} u^{v}-\Delta^{\mu \nu}\left(P_{0}+\Pi\right)+\pi^{\mu \nu}$, with $\varepsilon$ being the energy density, $P_{0}$ the thermodynamic pressure, $\Pi$ the bulk viscous pressure, and $\pi^{\mu \nu}$ the shear-stress tensor. We further introduced the projection operator $\Delta^{\mu \nu}=g^{\mu \nu}-u^{\mu} u^{\nu}$ onto the 3 -space orthogonal to the fluid velocity. We assume that the baryon number density and diffusion are zero at all space-time points and our metric convention is $g^{\mu \nu}=\operatorname{diag}(+1,-1-1-1)$.

The conservation laws and equation of state $P_{0}(\varepsilon)$ are not enough to determine the time evolution of $T^{\mu \nu}$. In order to do so, one still needs to provide the time-evolution or constitutive equations satisfied by $\Pi$ and $\pi^{\mu \nu}$. For this purpose, we employ relaxation-time equations derived from kinetic theory $[2,3]$, solved numerically using the MUsIC 
hydrodynamic simulation $[4,5]$. Explicitly, we solve

$$
\begin{aligned}
\tau_{\Pi} \dot{\Pi}+\Pi & =-\zeta \theta-\delta_{\Pi \Pi} \Pi \theta+\lambda_{\Pi \pi} \pi^{\mu v} \sigma_{\mu v}, \\
\tau_{\pi} \dot{\pi}^{\langle\mu v\rangle}+\pi^{\mu v} & =2 \eta \sigma^{\mu v}+2 \tau_{\pi} \pi_{\alpha}^{\langle\mu} \omega^{v\rangle \alpha}-\delta_{\pi \pi} \pi^{\mu v} \theta+\varphi_{7} \pi_{\alpha}^{\langle\mu} \pi^{v\rangle \alpha}-\tau_{\pi \pi} \pi_{\alpha}^{\langle\mu} \sigma^{v\rangle \alpha}+\lambda_{\pi \Pi} \Pi \sigma^{\mu v} .
\end{aligned}
$$

The above equations of motion include nonlinear terms that couple bulk viscous pressure to the shear-stress tensor and vice-versa. Recently, they were shown to be in good agreement with solutions of the $0+1$ Anderson-Witting equation in the massive limit [6]. The transport coefficients $\tau_{\Pi}, \zeta, \delta_{\Pi \Pi}, \lambda_{\Pi \pi}, \tau_{\pi}, \eta, \delta_{\pi \pi}, \varphi_{7}, \tau_{\pi \pi}$, and $\lambda_{\pi \Pi}$ are complicated functions of temperature. For strongly coupled fluids, such as the quark-gluon plasma created at the LHC, their values are not known. As a first step, we fix the parametric dependence of all transport coefficients on the shear viscosity, $\eta$, and bulk viscosity, $\zeta$, using formulas derived from the Boltzmann equation near the conformal limit [3].

The shear viscosity is assumed to be proportional to the entropy density, i.e., $\eta=a s$, where the parameter $a$ is fixed by fitting the data. In simulations using IP-Glasma initial conditions that include only dissipative effects from shear viscosity, one usually obtains $a=0.20-0.22$ at LHC energies [7, 8]. The bulk viscosity coefficient is parametrized as [3]

$$
\zeta=b \times \eta\left(\frac{1}{3}-c_{s}^{2}\right)^{2},
$$

where $c_{s}$ is the velocity of sound and $b$ a parameter that will also be adjusted to fit the data. Of course, the actual temperature dependence of each viscosity coefficient is not known for QCD matter. Nevertheless, the aforementioned parametrizations should be able to capture the relevant features of dissipation and will be enough for the purposes of this initial study.

The final state hadrons are produced following the usual Cooper-Frye procedure [10], in the same way as outlined in Ref. [8]. The only difference is that we have to specify the nonequilibrium correction to the hadron momentum distribution function due to bulk viscosity, in addition to the usual correction due to shear viscosity . Here, we employ the distribution derived from the Boltzmann equation using the relaxation time approximation, also used by Bozek et al [11],

$$
\begin{aligned}
\delta f_{\Pi}^{i} & =-f_{0 \mathbf{k}}^{i}\left(1 \pm f_{0 \mathbf{k}}^{i}\right)\left[\frac{1}{u_{\mu} k_{i}^{\mu}} \frac{m_{i}^{2}}{3}-\left(\frac{1}{3}-c_{s}^{2}\right) u_{\mu} k_{i}^{\mu}\right] \frac{\Pi}{C_{\Pi}} \\
C_{\Pi} & =\frac{1}{3} \sum_{i=1}^{N} m_{i}^{2} g_{i} \int d K_{i} f_{0 \mathbf{k}}^{i}\left(1 \pm f_{0 \mathbf{k}}^{i}\right)\left[\frac{1}{u_{\mu} k_{i}^{\mu}} \frac{m_{i}^{2}}{3}-\left(\frac{1}{3}-c_{s}^{2}\right) u_{\mu} k_{i}^{\mu}\right] .
\end{aligned}
$$

Above, $k_{i}^{\mu}, m_{i}$, and $g_{i}$ are the 4-momentum, mass, and degeneracy factor of the $i$-th hadron species, respectively, $N$ is the number of hadrons considered in the calculation, and $f_{0 \mathbf{k}}^{i}$ is the Fermi-Dirac or Bose-Einstein distribution function.

We note that the IP-Glasma initial conditions used in this work include the effect of nucleon-nucleon correlations in the initial state [9]. Such correlations were recently found to be important when simulating central and ultracentral collisions with the IP-Glasma model [8].

\section{Results}

We calculate the integrated elliptic flow coefficient $v_{n}\{2\}$ via the cumulant method. In order to compare with CMS data [13], we use a lower cut off of $0.3 \mathrm{GeV}$ when performing integrals in the transverse momentum, $p_{T}$. All our calculations were done using the parametrization of the lattice QCD equation of state by Huovinen and Petreczky [12]. The calculations with bulk viscosity were all performed in chemical equilibrium and with a freezeout temperature of $T_{F O}=140 \mathrm{MeV}$. The calculations with only shear viscosity were performed using the same parameters as in [8], with a chemical freezeout temperature of $T_{C F}=150 \mathrm{MeV}$ and $T_{F O}=103 \mathrm{MeV}$. In all figures, the shaded bands represent the statistical uncertainty.

In Fig. 1 we show the results for a given shear viscosity, $\eta / s=0.15$, and several values of $b=15,45$, and 75 . We see that increasing the value of bulk viscosity improves the agreement with the flow harmonics measured by CMS. In Fig. 1(b), we compare a simulation which includes only shear viscosity, with $\eta / s=0.22$, to another with $\eta / s=0.15$ 


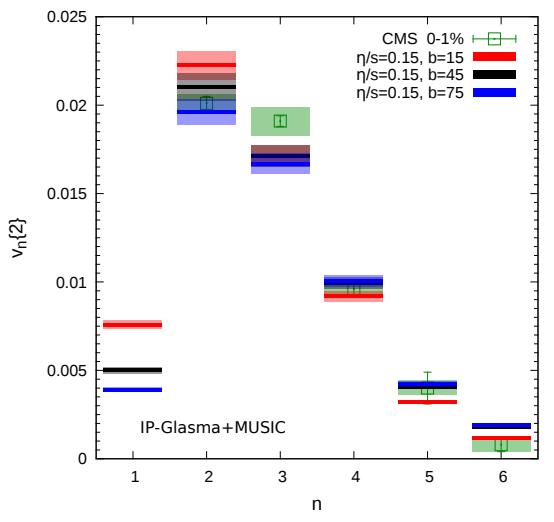

(a)

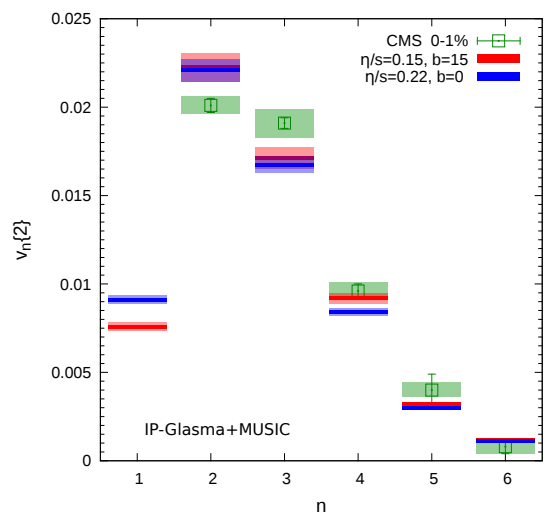

(b)

Figure 1. (Color online) Comparison of the flow harmonics, $v_{n}\{2\}$, calculated using IP-Glasma initial conditions, with with CMS data for the 0-1\% centrality class and for several values of the parameter $b$.

and $b=15$. Both calculations provide an almost equally good agreement with the flow harmonics measured by CMS, but with rather different values of shear viscosity. In this case, including bulk viscosity reduced the value of $\eta / s$ by $32 \%$, from 0.22 to 0.15 .

In Fig. 2(a) we show what happens when the parameter $b$ is fixed as $b=45$ and the shear viscosity is varied, taking the values $\eta / s=0.12,0.15$, and 0.18 . Note that the parametrization of bulk viscosity used in this work, Eq. (3), is linear in the shear viscosity, so keeping $b$ constant does not imply that the bulk viscosity is kept fixed. In this figure we see that the value of shear viscosity that provides the best overall agreement with the CMS data is $\eta / s=0.15$, which was the value already employed in the simulations discussed above.

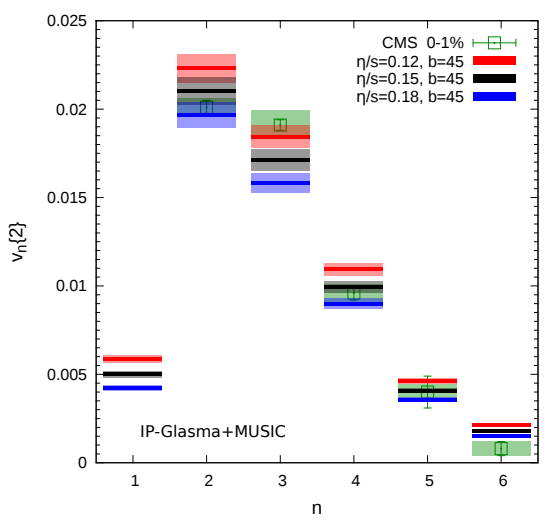

(a)

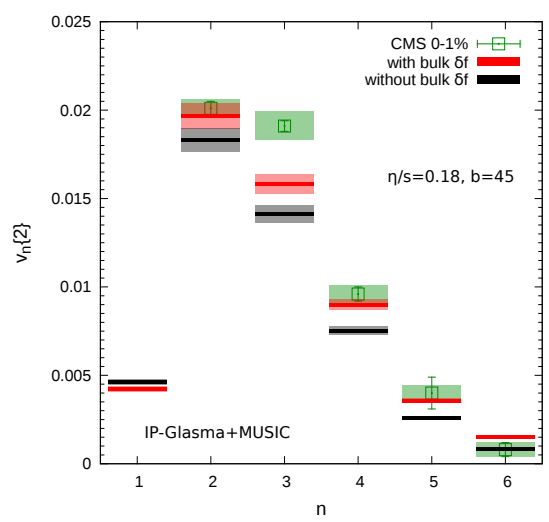

(b)

Figure 2. In the left panel we show the flow harmonics, $v_{n}\{2\}$, calculated using IP-Glasma initial conditions, for $b=45$ and several values of $\eta / s$ (left panel). In the right panel we show the effect of $\delta^{i} f_{\Pi}$ on $v_{n}\{2\}$.

In Fig. 2(b) the results for $\eta / s=0.18$ and $b=45$ are shown calculated with and without the bulk component of $\delta f, \delta^{i} f_{\Pi}$. In contrast to the shear correction, the bulk correction to the single particle distribution function used in this work lead to an increase in the flow harmonics. Such an increase was systematically stronger for higher harmonics: $v_{2}\{2\}$ is increased by $4 \%, v_{3}\{2\}$ by $8 \%, v_{4}\{2\}$ by $20 \%, v_{5}\{2\}$ by $57 \%$, and $v_{6}\{2\}$ by $117 \%$. Therefore, in order to properly describe higher harmonics, it is important to take into account the $\delta^{i} f_{\Pi}$ contribution. However, one should note that 
different momentum dependences for this quantity can provide rather different results.

Finally, we show the effect of bulk viscosity on the mean transverse momentum of pions, $\left\langle p_{T}^{\pi}\right\rangle$, in Fig. 3. Our calculations are compared to ALICE data [14] in the 0-5\% centrality class and, for this purpose, we used a lower cut-off of $0.12 \mathrm{GeV}$ when performing integrals in transverse momentum. The effect of bulk viscosity on $\left\langle p_{T}^{\pi}\right\rangle$ is not small and can be used to further restrict the size of the bulk viscosity coefficient. Here, we see that the best description of $\left\langle p_{T}^{\pi}\right\rangle$ happens when $b=20-40$, for the case where $\eta / s=0.15$. Note that the $\left\langle p_{T}^{\pi}\right\rangle$ has a strong dependence on $\delta^{i} f_{\Pi}$, so the optimal values we obtained can be modified if another $\delta^{i} f_{\Pi}$ is used.

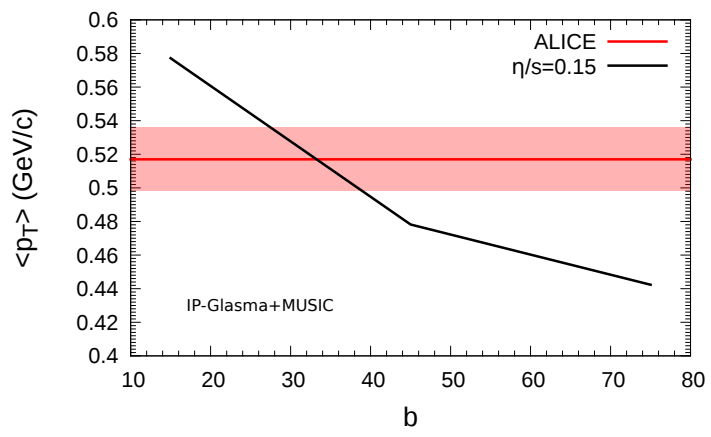

Figure 3. (Color online) The mean transverse momentum of pions, $\left\langle p_{T}^{\pi}\right\rangle$, for several values of $b$ compared to the ALICE data [14].

\section{Conclusions}

In this contribution we performed a preliminary study on the effects of bulk viscous pressure in ultracentral relativistic heavy ion collisions at the LHC. We found that bulk viscous pressure can have non-negligible effect on the flow harmonic coefficients in this centrality class. Furthermore, we showed that, at least for IP-Glasma initial conditions, the inclusion of bulk viscosity leads to a better description of the data. Although describing the anisotropy distribution of ultracentral events remains a challenge, we expect bulk viscosity to be a key ingredient in understanding such observables.

Acknowledgments: The authors thank H. Niemi and J. Jia for fruitful discussions. This work was supported in part by the Natural Sciences and Engineering Research Council of Canada, and by the U. S. DOE Contract No. DEAC02-98CH10886. G.S. Denicol acknowledges support through a Banting Fellowship of the Natural Sciences and Engineering Research Council of Canada, and C. G. acknowledges support from the Hessian Initiative for Excellence (LOEWE) through the Helmholtz International Center for FAIR (HIC for FAIR).

\section{References}

[1] B. Schenke, P. Tribedy and R. Venugopalan, Phys. Rev. C 86, 034908 (2012).

[2] G. S. Denicol, H. Niemi, E. Molnar and D. H. Rischke, Phys. Rev. D 85, 114047 (2012).

[3] G. S. Denicol, S. Jeon and C. Gale, accepted for publication in Phys. Rev. C, arXiv:1403.0962 [nucl-th].

[4] B. Schenke, S. Jeon and C. Gale, Phys. Rev. C 82, 014903 (2010).

[5] H. Marrochio, J. Noronha, G. S. Denicol, M. Luzum, S. Jeon and C. Gale, arXiv:1307.6130 [nucl-th].

[6] G. S. Denicol, W. Florkowski, R. Ryblewski and M. Strickland, arXiv:1407.4767 [hep-ph].

[7] C. Gale, S. Jeon, B. Schenke, P. Tribedy and R. Venugopalan, Phys. Rev. Lett. 110, 012302 (2013).

[8] G. S. Denicol, C. Gale, S. Jeon, J. -F. Paquet and B. Schenke, arXiv:1406.7792 [nucl-th].

[9] M. Alvioli, H.-J. Drescher and M. Strikman, Phys. Lett. B 680, 225 (2009).

[10] F. Cooper and G. Frye, Phys. Rev. D 10, 186 (1974).

[11] P. Bozek, Phys. Rev. C 81, 034909 (2010).

[12] P. Huovinen and P. Petreczky, Nucl. Phys. A 837, 26 (2010).

[13] S. Chatrchyan et al. [CMS Collaboration], JHEP 1402, 088 (2014).

[14] B. Abelev et al. [ALICE Collaboration], Phys. Rev. C 88, no. 4, 044910 (2013). 